Communications in Physics, Vol. 27, No. 4 (2017), pp. 339-344

DOI:10.15625/0868-3166/27/4/10836

\title{
DEPOLARIZATION PROPERTY OF CIRRUS CLOUDS OVER HANOI
}

\author{
TIEN PHAM MINH ${ }^{a, b}$, TUAN NGUYEN XUAN $^{c}$, TRUNG DINH VAN $^{c}$, MANH LE DUY $^{d}$, \\ AND HAI BUI VAN ${ }^{e}$ \\ ${ }^{a}$ Ho Chi Minh City Institute of Physics, Vietnam Academy of Science and Technology, \\ No 1 Mac Dinh Chi, Ben Nghe, District 1, Ho Chi Minh City, Vietnam \\ ${ }^{b}$ Graduate University of Science and Technology, Vietnam Academy of Science and Technology, \\ 18, Hoang Quoc Viet, Cau Giay, Ha Noi, Vietnam \\ ${ }^{c}$ Institute of Physics, Vietnam Academy of Science and Technology, \\ 10 Dao Tan, Ba Dinh, Hanoi, Vietnam \\ ${ }^{d}$ Institute of Research and Development, Duy Tan University, \\ No. 3 Quang Trung, Da Nang, Vietnam \\ ${ }^{e}$ Le Quy Don Technical University, 236 Hoang Quoc Viet, North Tu Liem, Hanoi, Vietnam \\ $\dagger$ E-mail: bvhaihsh@yahoo.com, bvhaihsh@mta.edu.vn
}

Received 10 October 2017

Accepted for publication 27 October 2017

Published 22 December 2017

\begin{abstract}
Cirrus cloud is one of the most commonly occurring cloud types globally. The clouds are found mainly to consist of floating ice crystals, which can reflect strongly incoming radiation. Lidar measurements provide an opportunity to study the microphysics and ice compositions of cirrus clouds. We have developed a polarization lidar system to measure the depolarization characteristics of the lower atmosphere over Hanoi. From our measurements of cirrus clouds over the period from 2010 to 2012, the depolarization ratio of cirrus clouds is found to be very high ranging from $20 \%$ up to $80 \%$, indicating large fraction of backscattering from ice crystals. We find a trend of increasing depolarization ratio with height and decreasing temperature. We also compare our lidar measurements with other results obtained by lidar technique in other regions of the world.
\end{abstract}

Keywords: lidar, atmosphere, depolarization.

Classification numbers: 42.68.Ay, 92.60.Ta.

(C)2017 Vietnam Academy of Science and Technology 


\section{INTRODUCTION}

Cirrus clouds, usually present in the upper troposphere, play an important role in the energy balance of the Earth's atmosphere [1,2]. These clouds consisting mainly of non-spherical ice crystals, typically cover about $30 \%$ of the Earth's surface and contribute to the radiation budget through the trapping of outgoing infrared radiation (greenhouse effect) and the reflection of incoming solar radiation (albedo effect) [2]. Therefore, the physical and optical properties as well as the location of cirrus clouds are important parameters to understand the radiation balance of the atmosphere. For cirrus clouds the physical parameters of primary concern are thickness, altitude, mean altitude and mid-cloud temperature. In addition, parameters related to microphysics of the clouds such as the shape, size and orientation of ice crystals play an important role in determining the cloud radiative properties [3-5].

Lidar (light detection and ranging) is a very powerful remote sensing technique to study the cirrus clouds. The backscattering signal from the clouds provides direct measurements of their main characteristics. Ice crystals present in the cirrus clouds are known to scatter strongly, producing significant depolarization. Therefore, a polarization lidar system can detect cirrus clouds and study the properties of crystal particles. At the Institute of Physics we have successfully developed a polarization lidar system to study the characteristics of aerosols and clouds over Hanoi $\left(21^{\circ} 2^{\prime} 0^{\prime \prime} \mathrm{N}, 105^{\circ} 51^{\prime} 0^{\prime \prime} \mathrm{E}\right)$, Vietnam. In this work we describe our lidar measurements over the period $2010-2012$ and the relation between depolarization properties with height and temperature of cirrus clouds.

\section{LIDAR MEASUREMENT}

\section{II.1. Polarization Lidar system}

The compact polarization lidar system developed for the present work as shown schematically in Fig. 1 uses the high power pulsed Nd:YAG laser (Brilliant Quantel, France) as transmitter. The laser delivers a pulse energy of $180 \mathrm{~mJ}$ at $532 \mathrm{~nm}$, a repetition rate of $10 \mathrm{~Hz}$ and a pulse duration of $5 \mathrm{~ns}$. The divergence of the laser beam is measured to be better than $1 \mathrm{mrad}$. The linear polarization direction of the laser beam can be manually rotated using a half wave plate mounted on a precision rotary stage.

The receiver is a diameter $25 \mathrm{~cm}$ Cassegrain telescope $(f / D=10)$. To reduce the background light we limit the field of view of the telescope to $1.5 \mathrm{mrad}$ during the lidar measurement by using a pinhole as spatial filter. We also use a high quality interference band pass filter with band width of $3 \mathrm{~nm}$ at wavelength of $532 \mathrm{~nm}$ to remove the background light. A polarization beam splitter is used to separate the backscattered signal into two channels of perpendicular polarization directions. The backscattered light in the two channels is detected by photomultiplier tubes R7400U configured in analog mode as shown in Fig. 1. The signal is then digitized using a high speed 12 bit ADC sampling at 20 MSPS. For each lidar measurement, the backscattering intensity profile is collected for 12000 laser pulses, equivalent to 20 minutes time interval.

The gain ratio of two polarization channels is the most important parameter in our depolarization measurements. To determine the gain factors, we rotate the half wave plate to determine the maximum and minimum intensities of the backscattering signal in both polarization channels for altitudes between $6-10 \mathrm{~km}$ where the air is known to be relatively free of aerosols, so the depolarization ratio should be the same as for air molecules. The gain ratio is then estimated by 


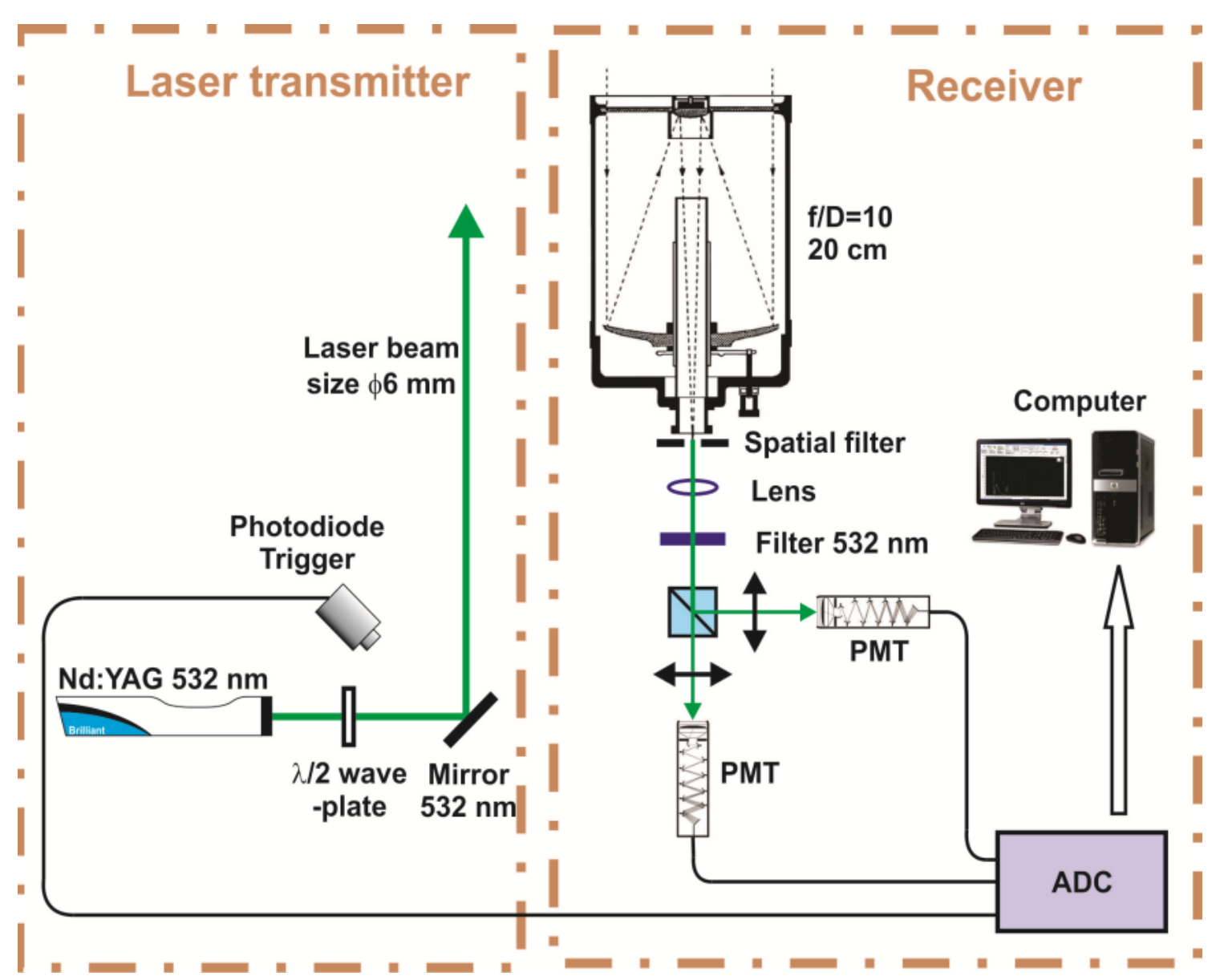

Fig. 1. Schematics of the polarization lidar system.

comparing the intensities measured for each polarization channel. After the gain calibration we check the depolarization fraction of clean air region and find a value of about $4 \%$, consistent with the theoretical expectation and laboratory measurements [5].

\section{II.2. Data analysis}

The depolarization $\delta(z)$ is definited as a ratio

$$
\delta(z)=\frac{P_{r s}(z)}{P_{r p}(z)}
$$

where, $P_{r p}(z)$ and $P_{r s}(z)$ are the integrated returned signal powers in parallel and perpendicular directions relative to the outgoing laser beam. $k_{1}$ and $k_{2}$ are the system factors of two depolarization channels, respectively. The lidar equations for parallel and perpendicular directions can be written as follows:

$$
P_{r p}(z)=\frac{k_{p} P_{t}}{z^{2}} \beta_{p}(z) \cdot \exp \left(-2 \int_{0}^{z} \tau_{p}(z) d z\right),
$$




$$
P_{r s}(z)=\frac{k_{s} P_{t}}{z^{2}} \beta_{s}(z) \cdot \exp \left(-\int_{0}^{z}\left(\left(\tau_{p}(z)+\tau_{s}(z)\right) d z\right),\right.
$$

where $P_{t}$ is the transmitted power, $\beta_{s}(z)$ and $\beta_{p}(z)$ are the volume backscattering coefficients at perpendicular and parallel directions, $k_{p}$ and $k_{s}$ are the system gain factors in parallel and perpendicular channels and $\tau(z)$ is the extinction coefficient of the atmosphere. As a result, we can express the depolarization ratio as follows:

$$
\delta(z)=\frac{\beta_{s}(z)}{\beta_{p}(z)}=\frac{P_{r s}(z) / k_{s}}{P_{r p}(z) / k_{p}}=\frac{\beta_{s}(z)}{\beta_{p}(z)} \exp \left(\int_{0}^{z}\left(\tau_{p}(z)-\tau_{s}(z)\right) d z\right)
$$

And generally $\tau_{p}(z)=\tau_{s}(z)$ then $\delta(z)=\frac{P_{r s}(z) / k_{s}}{P_{r p}(z) / k_{p}}=\frac{k_{p}}{k_{s}} \cdot \frac{P_{r s}(z)}{P_{r p}(z)}$.

The gain ratio $\frac{k_{p}}{k_{s}}$ is determinated as described in the previous section.

\section{RESULTS AND DISCUSSIONS}

In Fig. 2 we show the profile of depolarization ratio of a layer of cirrus cloud over Hanoi, Vietnam, on November, 2012. The depolarization ratio reached a peak value of $62 \%$ at the height of $14 \mathrm{~km}$ in the middle of the cirrus cloud layer. The high depolarization ratio in Fig. 2 clearly indicates that the cloud layer contains mainly highly non-spherical ice-crystals, because spherical particles are not expected to produce depolarization [5-8]. In addition, in the clear air regions below and above the cloud layer, the depolarization ratio is about $4 \%$, mainly due to the asymmetry of air molecules [5].

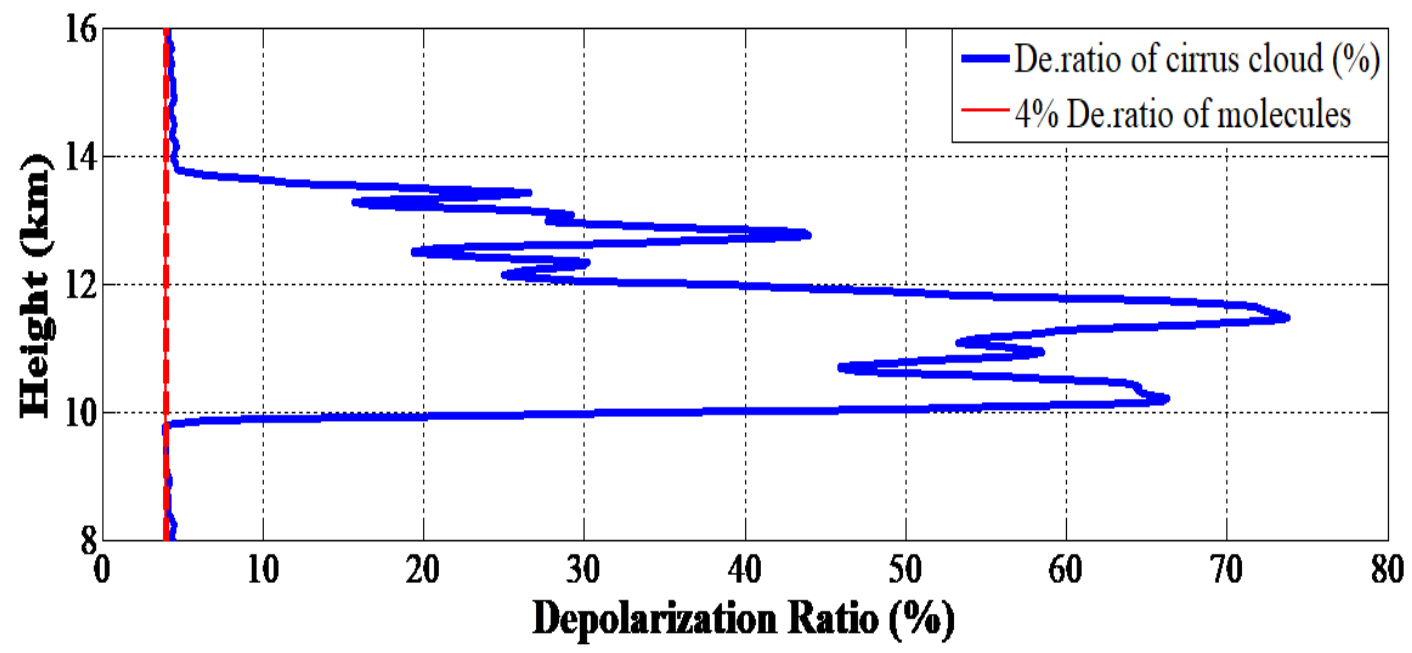

Fig. 2. Depolarization ratio profile of the cirrus cloud measured over Hanoi in June, $3^{\text {th }}, 2011$. 
In Fig. 3 we show the depolarization ratio of cirrus clouds with height. There is a tendency for the depolarization ratio to increase with height, similar to that found by [8] from observations of cirrus clouds over Northern Taiwan.

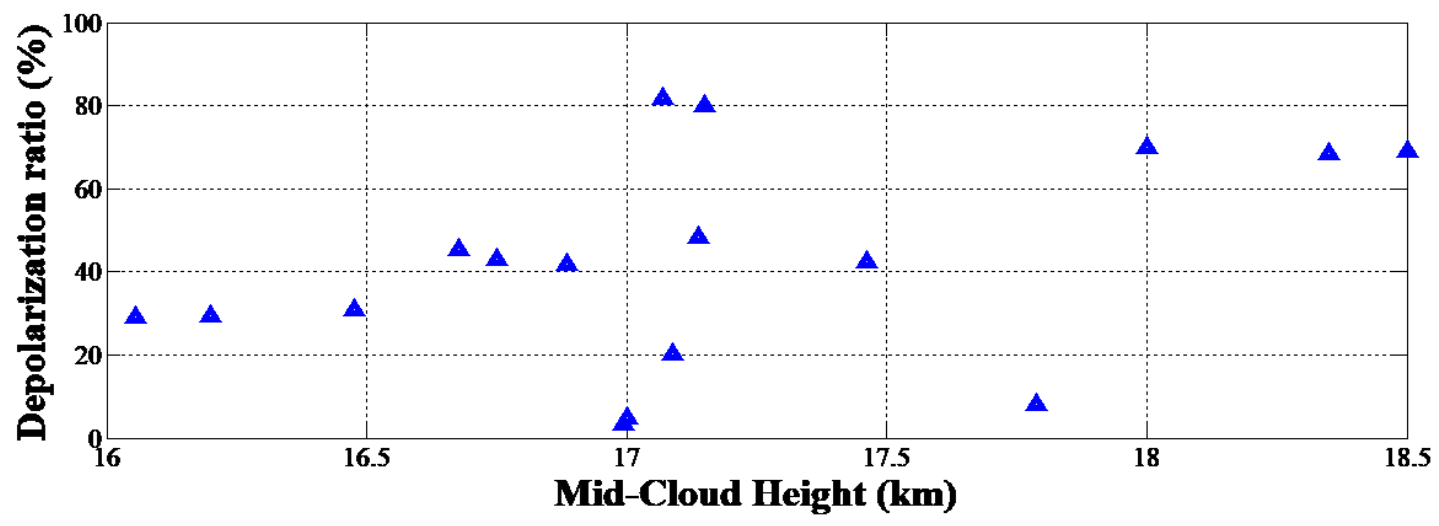

Fig. 3. Dep Depolarization ratio versus mid-cloud height of the detected cirrus clouds.

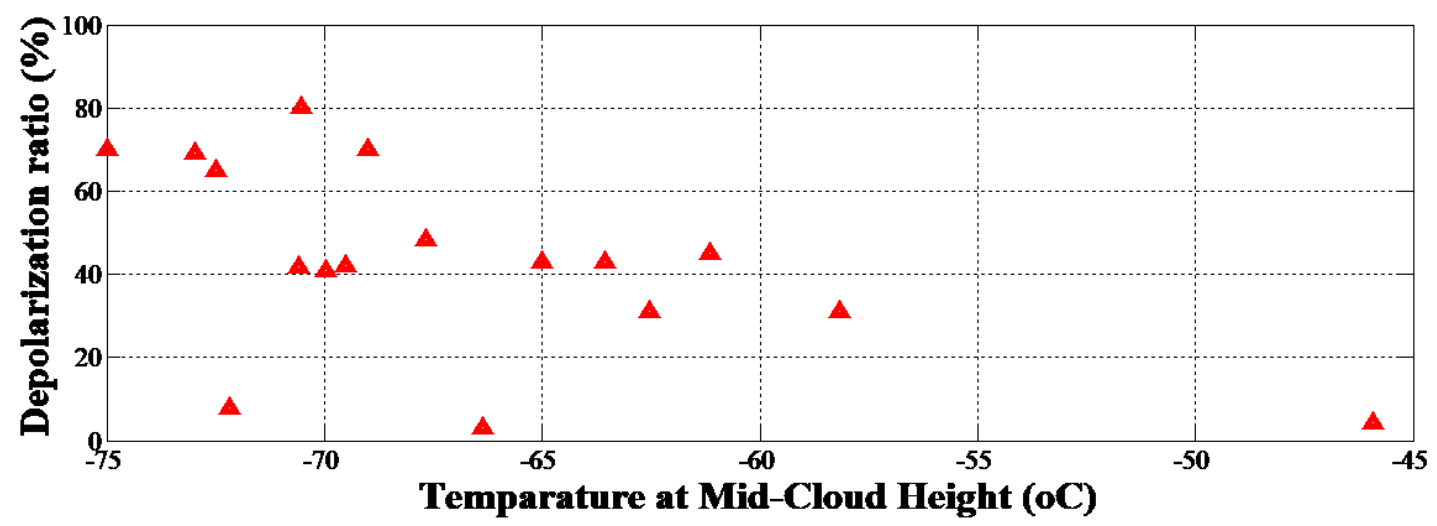

Fig. 4. Depolarization ratio versus mid-cloud temperature of the detected cirrus clouds.

In the troposphere the temperature decreases steadily with height, the depolarization ratio also exhibits an increasing trend with lower temperature at the location of cirrus clouds as shown in Fig. 4. In this case the data for cloud temperature is compiled from in-situ radiosonde measurements. We also note that the trend of increasing depolarization ratio with decreasing temperature has been seen earlier by [11]. They suggest that the trend appears as a result of the occurrence of complex ice crystal aggregates at low temperatures. The high depolarization values, up to $80 \%$, for cirrus clouds over Hanoi as measured by our polarization lidar system are entirely consistent with the results reported earlier for other regions of the world such as Taiwan [8], China [9], India [10], and North America [11]. 


\section{CONCLUSIONS}

In this paper we have presented the long term observations of the high cirrus clouds over Hanoi by a polarization lidar system. Our measurements of the depolarization ratio during a long period of time, from 2010 to 2012 indicate highly depolarized backscattering from the cirrus clouds, ranging from $20 \%$ up to $80 \%$, consistent with the presence of ice crystals in the clouds. Combining lidar and radiosonde data we show that the depolarization ratio increases with the cloud height and the decreasing temperature of the cirrus layer in the atmosphere over Hanoi, Vietnam. These results are consistent with the previously reported depolarization measurements in other parts of the world.

\section{ACKNOWLEDGMENT}

We acknowledge the financial support of the National Foundation for Science and Technology Development (Nafosted) under grant number 103.99-2014.82.

\section{REFERENCES}

[1] K.-N. Liou, Monthly Weather Review 114 (1986) 1167.

[2] P. Seifert, A. Ansmann, D. Müller, U. Wandinger, D. Althausen, A. Heymsfield, S. Massie and C. Schmitt, $J$. Geophys. Res.: Atmospheres 112 (2007) D17205.

[3] G. Beyerle, H.-J. Schäfer, R. Neuber, O. Schrems and I. McDermid, Geophys. Res. Lett. 25 (1998) 919.

[4] S. A. Bischoff, P. O. Canziani and A. E. Yuchechen, Int. J. Climatology 27 (2007) 189.

[5] R. M. Measures, Laser Remote Sensing, Wiley-Interscience, 1992.

[6] N. X. Tuan, D. Van Trung, D. D. Thang and B. Van Hai, Gated - photomultiplier tube for uses in lidar to study the upper atmosphere, The Proceedings of the Second Academic Conference on Natural Science For Master and Phd Students from Cambodia, Laos, Malaysia \& Vietnam, 2011, p. 393; ISSN: 978-604-913-088-5.

[7] B. V. Hai, D. V. Trung, N. X. Tuan, D. D. Thang and N. T. Binh, Comm. Phys. 22 (2013) 357.

[8] W.-N. Chen, C.-W. Chiang and J.-B. Nee, Applied Optics 41 (2002) 6470.

[9] K. Sassen and S. Benson, Journal of the Atmospheric Sciences 58 (2001) 2103.

[10] M. Min, P. Wang, J. R. Campbell, X. Zong and J. Xia, Advances in Atmospheric Sciences 28 (2011) 653.

[11] V. Sivakumar, Y. Bhavanikumar, P. Rao, K. Mizutani, T. Aoki, M. Yasui and T. Itabe, Radio Science 38 (2003) 1094. 